\title{
A Content Based Image Retrieval System using homogeneity Feature extraction from Recency-based Retrieved Image Library
}

\author{
Seema H.Jadhav ${ }^{1}$, Dr.Shah Aqueel Ahmed ${ }^{2}$

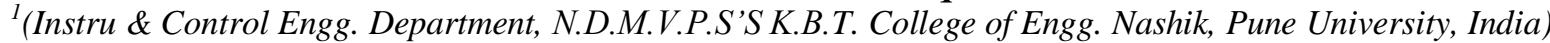 \\ ${ }^{2}$ (EIE Department, Royal Institute of Science \& Technology, Damergidda, A.P, JNTU University, India)
}

\begin{abstract}
Recently, Content Based Image Retrieval (CBIR) plays a significant role in the image processing field. The construction of large datasets has been facilitated by the developments in data storage and image acquisition technologies. In order to manage these datasets in an efficient manner development of suitable information systems are necessary. Based on image content, CBIR extracts images that are relevant to the given query image from large image databases. Images relevant to a given query image are retrieved by the CBIR system utilizing either low level features such as shape, color, texture and homogeneity or high level features such as human perception. Most of the CBIR systems available in the literature extract only concise feature sets that limit the retrieval efficiency. In this paper, additional feature extraction such as homogeneity based feature extraction is used along with color, shape and texture feature extraction to extract the query image from the database images as well as from the RRI library and also to store the retrieved images in the RRI library. The proposed CBIR technique is evaluated by querying different images and the retrieval efficiency is evaluated by determining precision-recall values for the retrieval results.
\end{abstract}

Keywords: Content Based Image Retrieval, homogeneity feature, High level feature, Image Segmentation, $k$ mean algorithm, Low level feature.

\section{INTRODUCTION}

Visual information has been used in a wide-range of fields as a result of the rapid development of digital imaging and networking technologies. The necessity to efficiently retrieve such information from databases has increased the interest in image content based image retrieval techniques. Most of the approaches which have been proposed in the past for image retrieval use Content-Based Image Retrieval (CBIR) methods which retrieve images by means of keywords or image contents [1]. In image processing, CBIR is considered as an important domain because of its diverse applications in internet, multimedia, medical image archives, satellite imaging [16], commerce, government [9], and academia and crime prevention [6]. Color, Shape and texture are important cue in extracting information from images; these histograms are widely used in content based image retrieval [12]. Color and texture contain important information but, for instance, two images with similar color histograms can represent very different things. Therefore the use of shape-describing features is essential in an efficient content-based image retrieval system. Although shape description has been intensively researched, there exists no direct answer as to which kind of shape features should be incorporated into such a system [13]. Most traditional and common methods of image retrieval utilize some method of adding metadata such as captioning, keywords or descriptions to the images so that retrieval can be performed over the annotation words.

Images are indexed by CBIR using the image features like color, shape and texture with minimal human involvement. CBIR compares the features of the images present in the database with that of the query image for retrieving relevant images [4] from the image data base for a specified query image [5]. CBIR has been developed into a popular field of research. Determining the finest solution for efficient retrieval of multimedia data and utilization of storage space has been attempted by several research domains [2]. Each image is represented in CBIR using image features such as color, texture and shape [8]. Images are retrieved using the relevance that is computed based on the similarity of the features between them and the query image [3] [11].

In content based image retrieval systems, color is one of the vital and widely used visual features. Color histogram is extensively used as a representation technique. It captures the number of pixels having specific properties and it is statistically described as the combined probabilistic properties of diverse color channels (such as red, green and blue) [7]. Texture is an inherent property of all surfaces and signifies the visual patterns of homogeneity. Important information related to the structural arrangement of the surface, such as clouds, leaves, bricks, fabrics, etc are present in it. The relationship between the surface and its neighboring environment are also represented by it. In short, the distinctive physical composition of a surface is described by this feature [17]. Texture determination is perfectly appropriate for medical image retrievals [10]. CBIR utilizes some texture features including entropy, energy, contrast and homogeneity [9]. 
Shape is one of the primary low level image features in content-based image retrieval. There are generally two types of shape representations: region based and contour based [14]. Region based systems typically use moment descriptors that include Geometrical moments, Zernike moments and Legendre moments. The Zernike basis functions satisfy the orthogonal property, implying that the contribution of each moment coefficient to the underlying image is unique and independent, (i.e.). No redundant information overlapped between the moments [15]. The characteristic surface configuration of an object; an outline or contour can be indicated by the term, shape. By its outline, it enables an object to be distinguished from its surroundings. Boundary based and region based are two general categories of shape representation [17]. The CBIR systems which, employ visual features assumes the existence of a mapping between the low level visual features and the high level image semantics $[19,20]$ i.e., they assume that if visual content of the images are identical then their semantic content will also be identical. The term "semantic gap" was later introduced by researchers $[18,19$, and 20] because they did not agree with this assumption. The term semantic gap signifies the disparity between the low-level visual features and the high-level image semantics. In [18], Semantic gap is considered as the loss of information that occurs when an image is represented by its features [19].

CBIR system extracts the images that are appropriate to the specified query image based on the image content. The feature sets that limit the retrieval efficiency is been extracted by a majority of the CBIR systems existing in the literature. The proposed CBIR system using homogeneity feature extraction effectively retrieves the images relevant to the query image from the data base as well as from the RRI library when compared to the other CBIR system.

\section{PROPOSED METHODOLOGY}

Content based image retrieval plays a significant role in many of the fields such as medical imaging, education, surveillance applications and more. In order to retrieve image based on the given query image, either low level or high level features of the database may be used. In order to extract and retrieve the image an effective mechanism is needed. In our proposed system, we use a recently retrieved image library for the retrieval of the system which increases the precision of the retrieval in the proposed system. The feature extraction and retrieval phase are the two steps we considered in our proposed work. The figure 1 shows the entire process of our proposed system.

\subsection{Feature Extraction}

In this feature extraction process, the low level features shape, color, texture and homogeneity are retrieved for the query image from the database and also from recently retrieved image library. The feature extractions are classified in to low level feature extraction and high level feature extraction. Let $X$ be an image of size $P \times Q$ has related images which are to be retrieved and firstly its low level and then high level features are extracted. The shape, color, texture and homogeneity feature extraction are detailed in the following sections,

\subsubsection{Low Level Feature Extraction}

The shape, color, texture and homogeneity features are the low level features used in this CBIR for retrieval. In the feature extraction process, the low level features shape, color, the texture and homogeneity features are extracted for the query image from the database images and also from recently retrieved image library. After the completion of the feature extraction, the query image features compared with the database image features and recently retrieved image library. Hence the images which have similar low level features are retrieved. The subsequent sections detail the aforesaid process 


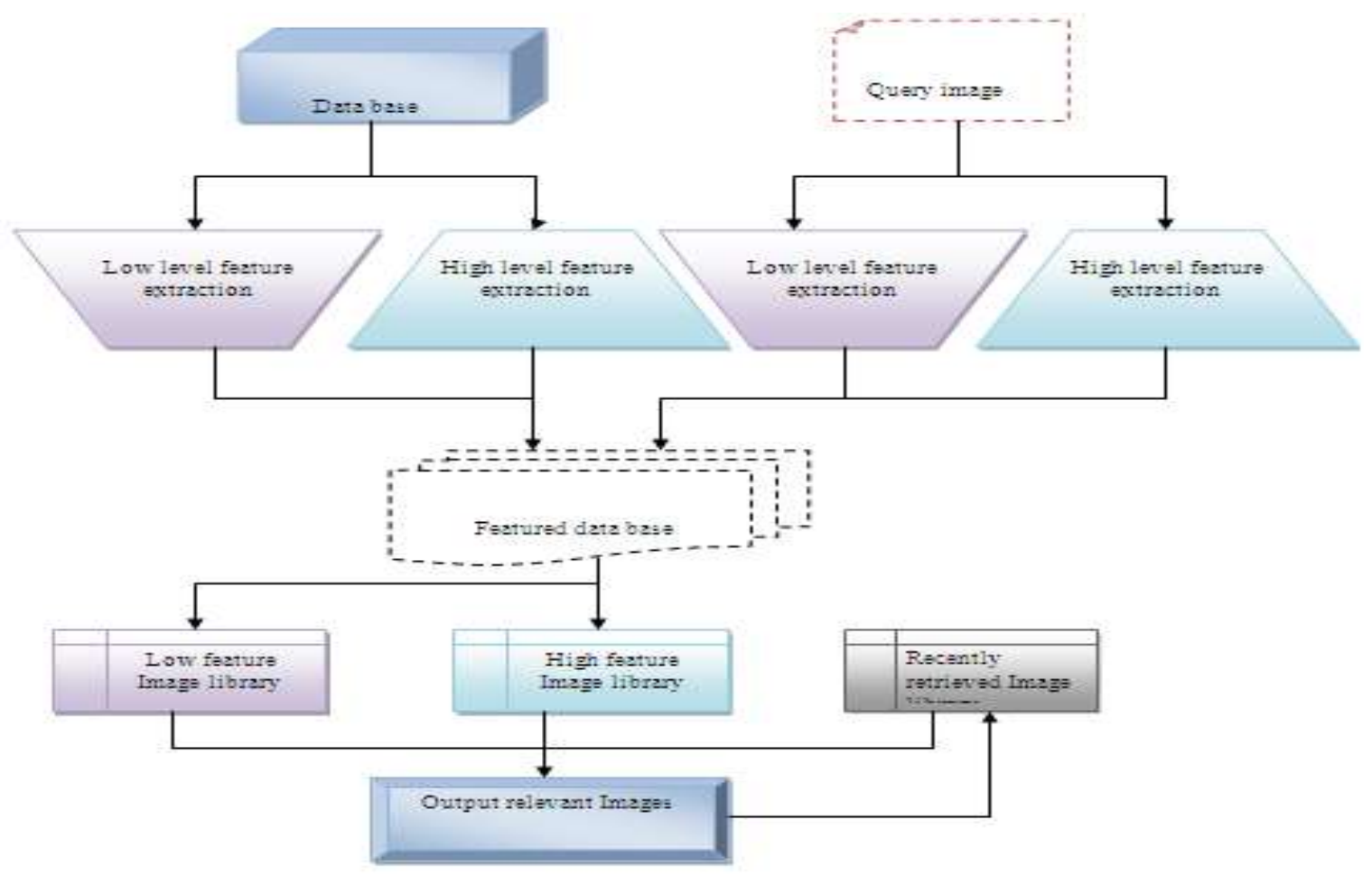

Figure 1: Process of the Proposed System

\subsubsection{Shape feature extraction}

Shape is an important visual feature and also it is one of the primitive features for image content explanation. Let $X$ be the image database, which contains images of dimension $P \times Q$. For the filterization process, the image $X$ is converted to gray scale $X_{g}$ from RGB color space. Let, $X_{R} X_{G}, X_{B}$ be the $R, G, B$ weights of the image $X$ then,

$: \mathbf{X}_{\mathbf{g}}=\mathbf{0 . 2 9 8 9} * \mathbf{X}_{\mathbf{R}}+\mathbf{0 . 5 8 7 0} * \mathbf{X}_{\mathbf{G}}+\mathbf{0 . 1 1 4 0} * \mathbf{X}_{\mathbf{B}}$

The equation (1) which is known as the Craig's formula is applied for the conversion of RGB to gray scale image and then the mean filter is applied on the converted gray scale image $X_{g}$ for the removal of noises. The mean filter smoothens the image data, thus the noise has been eliminated. Using the grey level values, this filter performs spatial filtering on each individual pixel in an image in a square or rectangular window surrounding each pixel. And then the filtered image is clustered to identify the various regions in the image.

This can be discovered by identifying groups of pixels that have similar gray levels, colors or local textures utilizing clustering in the image analysis. Clustering is a method of grouping data objects into different groups, such that similar data objects belongs to the same group and dissimilar data objects belongs to different clusters. For clustering process, numerous techniques exist and among them a vital role is played by the k means clustering method. For many practical applications, the k-means method has been shown to be effective in producing good clustering results. In this work, we use k means clustering to identify the various regions in the image. The denoised image $X_{g}$ is then clustered by means of k means clustering. The input of k means clustering involves Denoised image $X_{g}$ with $P \times Q$ pixels. The steps involved in the k means clustering process is as follows

At first step arbitrarily select the $K$ data items from the input as initial centroids and repeat the steps. Assign the remaining data items apart from the selected initial centroids to the cluster $K$, which has the closest centroids. Calculate the new centroids for each cluster until convergence occurs. After applying the $\mathrm{k}$ means clustering algorithm, the clustered regions of the denoised image $X_{g}$ is identified. Then, utilizing canny edge detection algorithm the diverse edges present in the clustered regions of the image $X_{g}$ are identified. The steps performed by the canny edge detection algorithm are shown below 
1. Smoothing: The k clustered regions of the image $X_{g}$ are blurred in order to remove the noises

2. Finding gradients: Then the edges of the clustered regions are marked wherever the gradients of the image possesses large magnitudes.

3. Non-maximum suppression: Only the local maxima of the clustered regions in the denoised image $X_{g}$ should be marked as edges.

4. Double thresholding: Potential edges of the clustered regions of the image $X_{g}$ are determined by a thresholding process.

5. Finally, the edges of the diverse clustered regions have been determined by suppressing all the edges which are not connected to a very certain edge.

Hence the edges of the clustered $\mathrm{k}$ regions are tracked and then the edges are smoothed for sharpening, in order to remove the number of the connected components that occur unnecessarily in the clustered regions. Therefore the diverse shapes which exist in the image $X_{g}$ are extracted and the shape feature is retrieved from the image $X_{g}$. Subsequently, the shape feature of the diverse images existing in the database are also extracted and stored as a shape feature vector set $S_{f}$

\subsubsection{Color feature extraction}

The problem of decomposing images into regions that is semantically uniform. Since images themselves provide only semantically poor information, image segmentation is essentially and applicationoriented problem that demands either strong intervention of human experts or application specific solutions [105]. The contour let transform is utilized to compute two essential features energy and standard deviation from the database images. At first the input image is re-sampled and then the image is converted into a gray-scale image. After that contour let transform is applied on the converted gray scale image [25]. Most of the images were rich in color and in our proposed technique different objects are segmented on the basis of colors. At first the number of different colors present in the image is reduced to 128 by using our proposed segmentation techniques. The LGB vector quantization algorithm is used in our technique in order to obtain the set of different colors which will represent image colors with respect to mean square error. The local contrast color value $\beta_{x \times y}$ as follows.

$$
: \quad \beta_{\mathbf{x} \times \mathbf{y}}=\frac{\left\|\kappa_{\mathbf{x y}}-\bar{\kappa} \mathbf{x y}\right\|}{\|\bar{\kappa} \mathbf{x y}\|}
$$

Where, $\bar{\kappa}_{x y}$ is the average of a color in the small neighborhood around $\kappa_{x y}$. The pixel $X_{x, y}$ is considered as an edge if its contrast exceeds a predefined value threshold $\lambda$. Next by distinguishing between the different uniform regions, texture areas, and contour points, we use a sliding window to estimate the several characteristics of the image such as mean $\eta$ and variance $\vartheta$ of edge density of each pixel. The smoothing algorithm usually is the average of the uniform and textured regions. The smoothed and color restored images is then subjected to mean shift color segmentation algorithm for segmentation

\subsubsection{Texture and Color intensity level Extraction}

The numerous common textures consist of small textons that are usually in very large number is understood as isolated objects. Here the texture features are extracted using the gray level difference method (GLDM) and the elements are placed more or less regularly or randomly. In this gray level difference method, diverse images are created in the four directions and then a feature vector is generated by linear zing the gray level histograms of these four new images. The texture features extraction is performed on the basis of the following Creation of different images. In the four directions i.e., north-east, north-west, south-east and southwest, the difference images are created. Then the probability distribution function is created from the gray level histograms of these four new images. The probability distribution function is created from the cumulative sum of the gray level histograms of these four new images. Likewise Color histogram features of the segmented objects are retrieved using the non linear diffusion algorithms. This color histogram is determined for all the images and it is stored as a color feature $\operatorname{set} C_{f}$. 


\subsubsection{Homogeneity feature extraction}

Let ' $X$ ' be an image of size $P \times Q$ has related images which are to be retrieved and firstly its low level and then high level features are extracted. The feature extraction using low level and high level feature extraction process is done by using the homogeneity feature extraction. The homogeneity feature extraction is used and it returns a value that measures the closeness of the distribution of elements in the GLCM to the GLCM diagonal. At first we have to create a gray level co-occurrence matrix and then the feature extraction process was carried out using our proposed extraction method.

Here, the steps we used to extract the feature from an image by using homogeneity feature extraction as follows Step 1: Create a gray-level co-occurrence matrix

Step 2: The gray-level co-occurrence matrix (GLCM) was created by calculating that how often a pixel with the gray level values $\mathrm{P}$ occurs in a specific spatial relationship to a pixel with the value $\mathrm{Q}$. The spatial relationship is derived from the pixel of interest and from the pixel to its immediate right but it specifies other spatial relationships between the two pixels.

Step3: After creating the GLCMs, we can extract features from them using the gray-level co-occurrence matrix function and homogeneity feature extraction is used.

Step 4: In homogeneity feature extraction the homogeneity returns a value that measures the closeness of the distribution of elements in the GLCM to the GLCM diagonal. Therefore it measure the closeness of the distribution of the element

Step 5: The homogeneity feature extraction process is done by using the formula as follows,

$$
: \mathbf{H}_{\mathbf{f}}=\sum_{\mathbf{P , Q}} \frac{\mathbf{f}(\mathbf{P}, \mathbf{Q})}{\mathbf{1}+|\mathbf{P}-\mathbf{Q}|}
$$

The feature extraction process is done by using homogeneity feature extraction process and by computing the process the extracted features are stored as $H_{f}$. Therefore the extracted low level and high level features are stored in the featured data base for retrieving images.

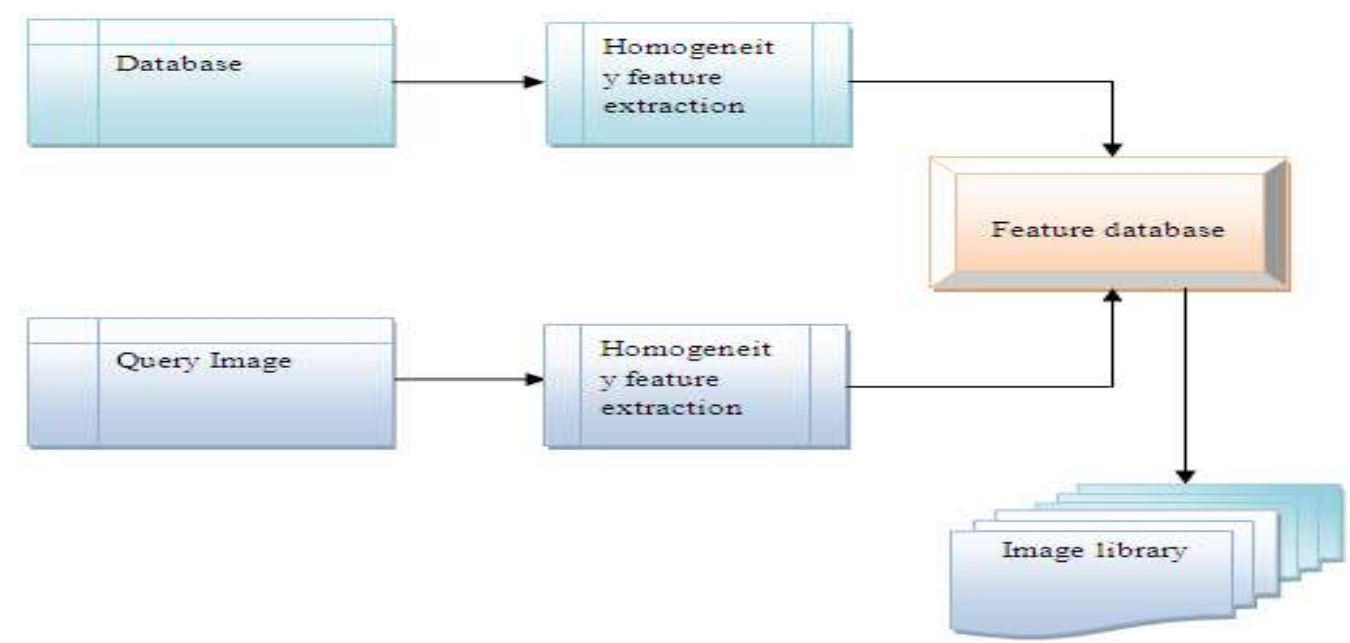

Figure 2: Homogeneity Based Feature Extraction

\subsection{High Level Feature Extraction}

This CBIR is based on the query keyword which is a high level feature in human understandable form. Relevant images are retrieved by this CBIR utilizing the keyword retrieved. Each image is indexed with its semantic meaning so that they could be identified by the CBIR.

\subsection{Retrieval Phase}

The query image is match up to the images in the database for image retrieval. After the extraction of images using shape, color, texture and homogeneity feature extraction, the extracted images are stored in the feature database. A semantic name is given to all the images, which is stored in the database, are also one of the high-level features of the image. The relevant images which satisfies the low level feature of the query images is retrieved first and stored in low feature image library and then images which satisfies the high level feature are extracted and stored in high feature image library. Based on the similarity of images which exists in low-level library, high-level library, and also in Recency-based retrieved image library (RRI library) related to the query image are retrieved. The recovered relevant images are stored with syntactic name index in RRI library for 
future reference. Therefore, the proposed CBIR technique using homogeneity feature extraction is evaluated by querying different images and the retrieval efficiency is effective.

\section{Experimental Results}

The proposed CBIR system was implemented in the working platform of MATLAB (version 7.10). The proposed system was assessed with different query images and the relevant images are obtained from the image database as well as RRI library. At first the initially images are segmented based on color subsequently shape features, texture, color histogram and homogeneity features were extracted from every image in the system database also every image has its semantic name as index name. The features extracted for query image and relevant images were subsequently found in the database as well as RRI library using the extracted features during the retrieval process. Therefore, the low level features as well as high level features are extracted and then the images relevant to the low level features and the images relevant to the high level features are retrieved separately. Then images, which are satisfying both the high level and low-level features, are selected from both the outputs retrieved using high and low level features and from the RRI Library. Finally, the obtained results are stored into the Recently Retrieved Library. The efficiency of the proposed system increases for each successive retrieval. The efficiency of the proposed system increases for each successive retrieval. The fig 3(b) and (c) are the color wise segmented image and extracted shape features of the query image in fig3(a).; fig3 (d) and (e) are the relevant images which are obtained using high level and low level features respectively. Fig3 (f) is the output of the proposed CBIR system. Thus, the process and consequent results of the proposed system using the same type of query image in the second retrieval and third retrieval are shown in fig4 and fig5 respectively.

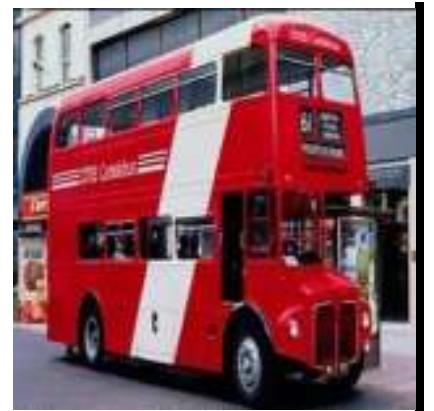

(a)

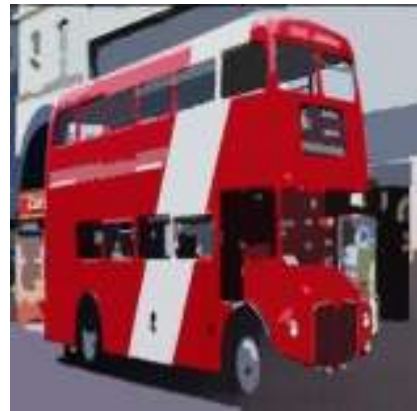

(b)

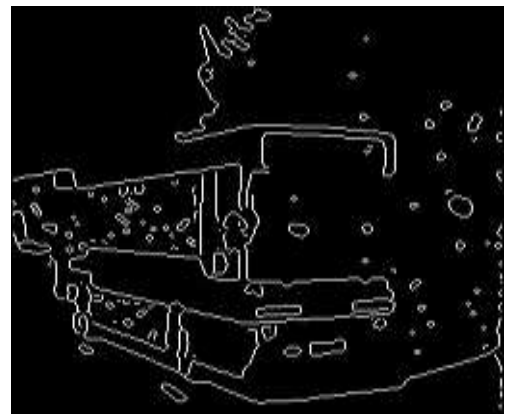

(c)

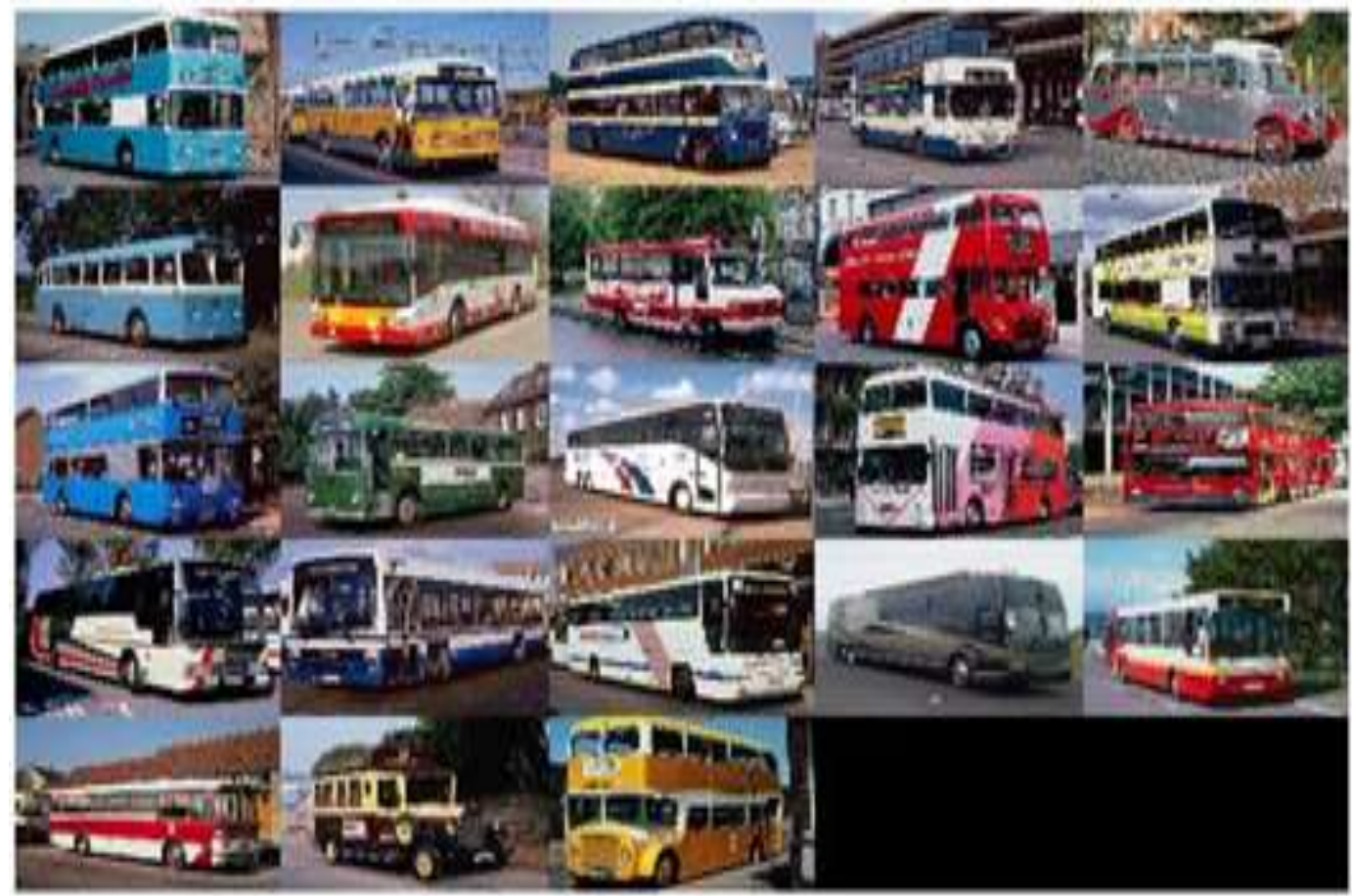

(d) 


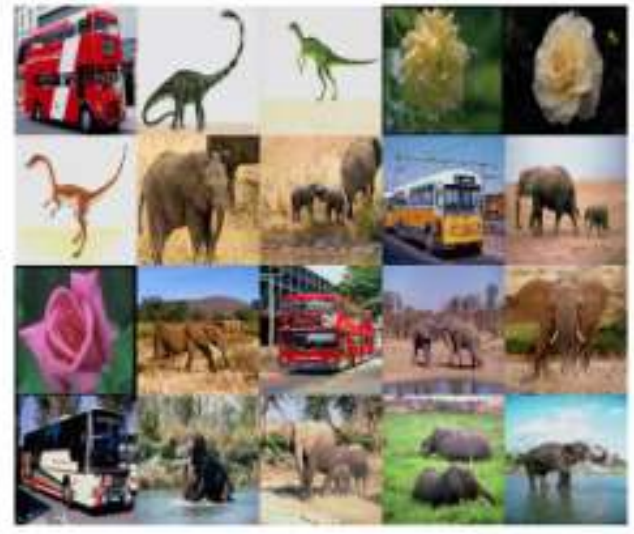

(e)

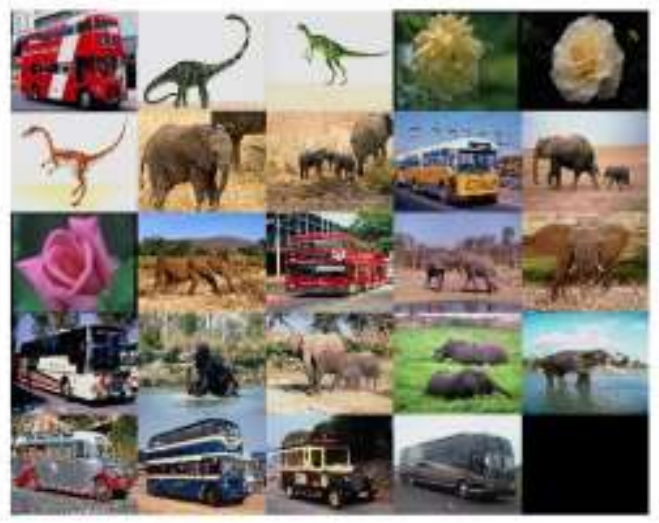

(f)

Figure-3: Process of First Retrieval (a) Query image (b) Color segmented image (c) Extracted shape feature (d) high level feature based retrieved output (e), Low level feature based retrieved output. (f)Output of first retrieval

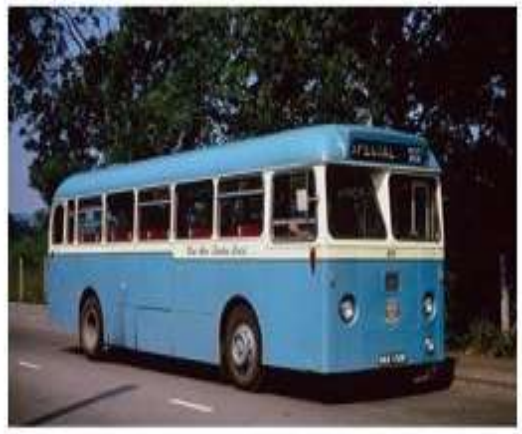

(a)

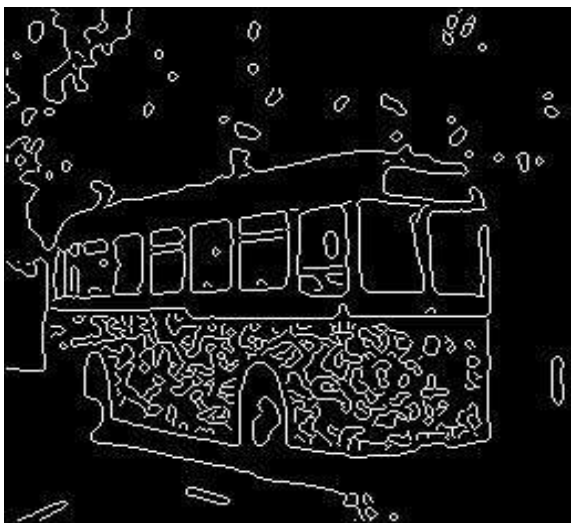

(c)

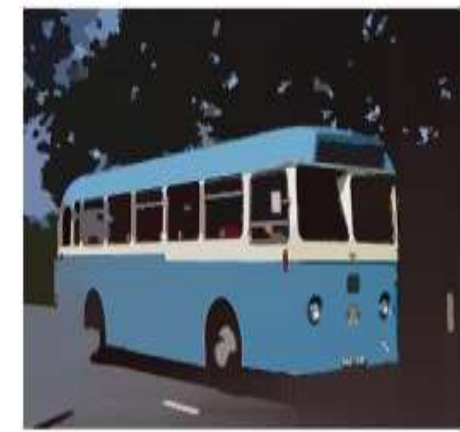

(b)

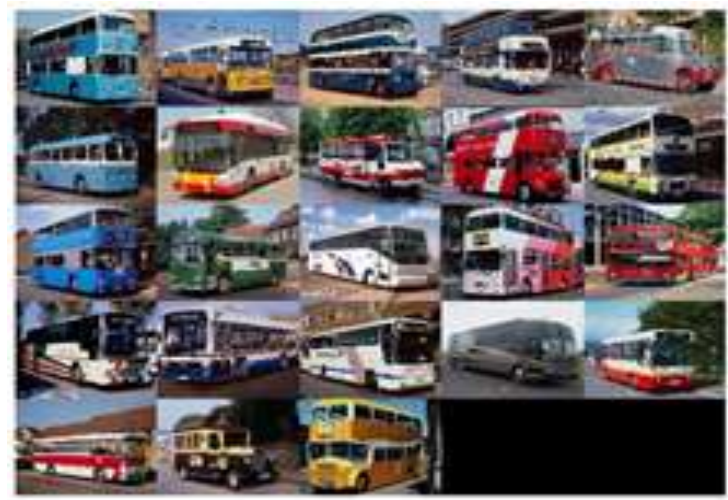

(d) 


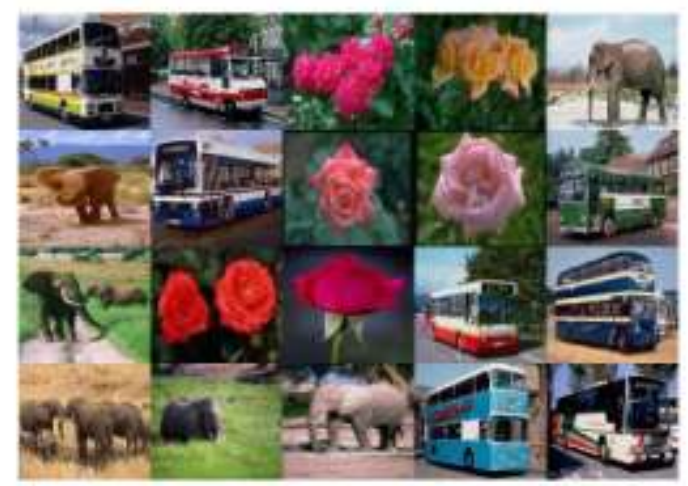

(e)

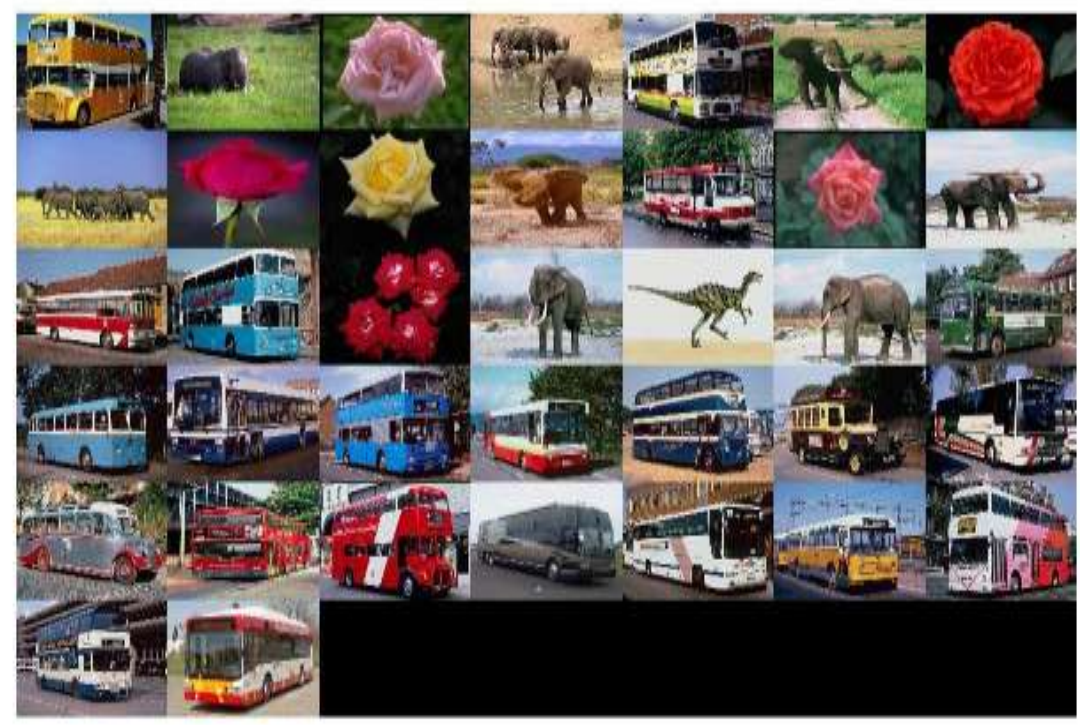

(f)

Figure-4: Process of Second Retrieval (a) Query image (b) Color segmented image (c) Extracted shape feature (d) high level feature based retrieved output (e), Low level feature based retrieved output. (f) Output of second retrieval

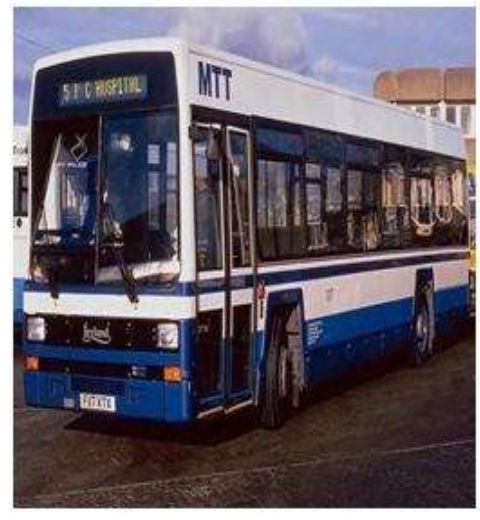

(a)

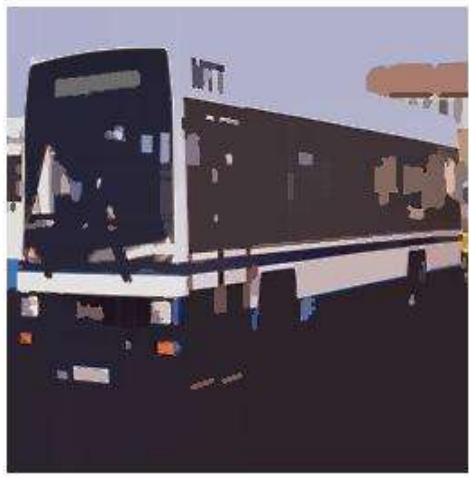

(b) 


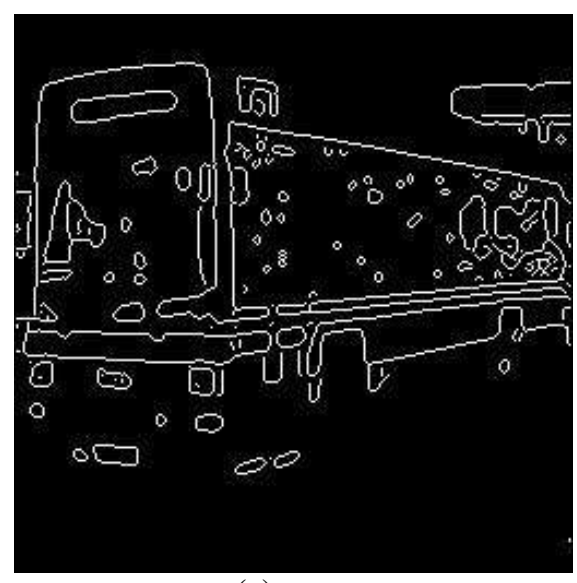

(c)

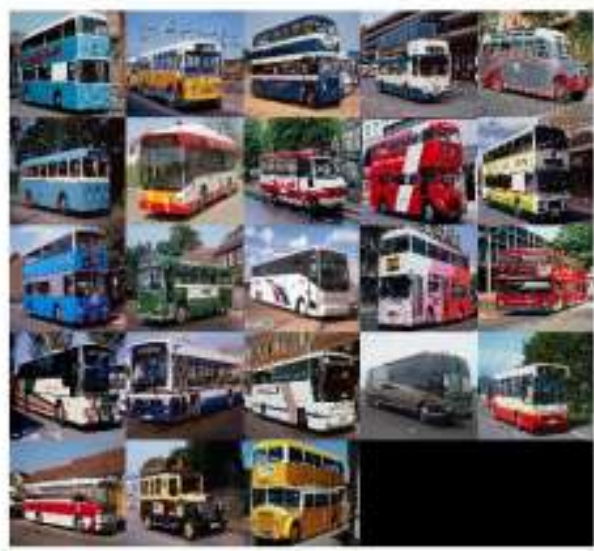

(d)

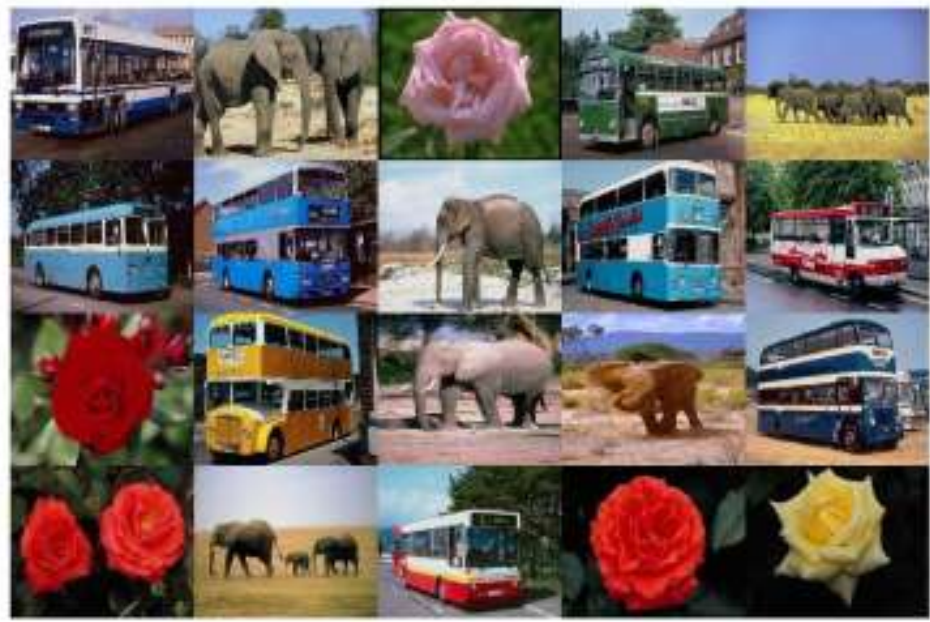

(e)

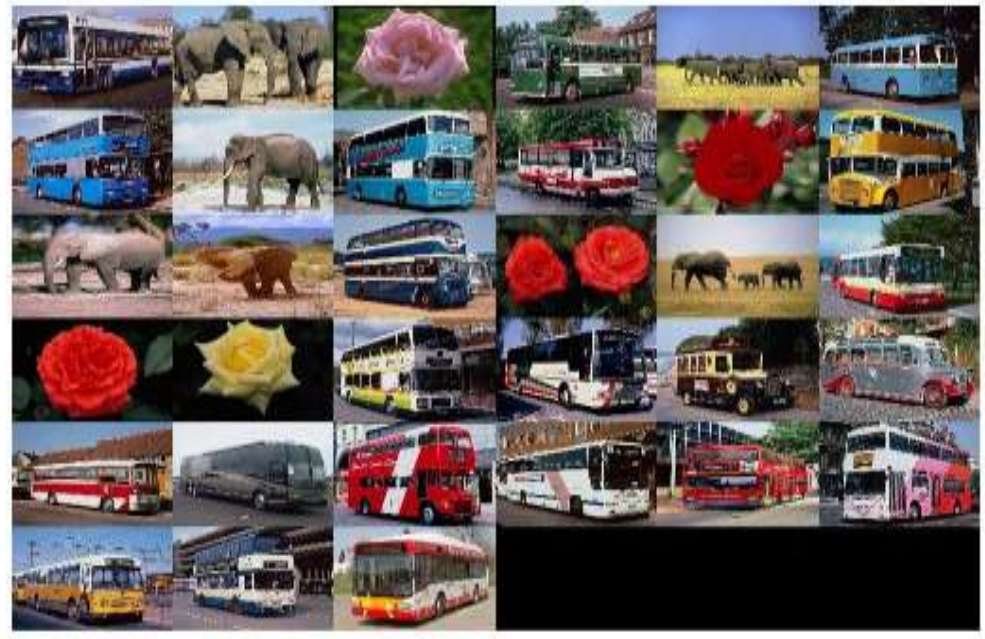

(f) 
Figure-5: Process of Third Retrieval (a) Query image (b) Color segmented image (c) Extracted shape feature (d) high level feature based retrieved output (e) Low level feature based retrieved output (f) Output of third retrieval.

Table 1: shows the comparison of number of images retrieved in our proposed and existing method

\begin{tabular}{|l|c|c|}
\hline $\begin{array}{c}\text { Number of } \\
\text { iterations }\end{array}$ & $\begin{array}{c}\text { Number of images retrieved } \\
\text { in our proposed method }\end{array}$ & $\begin{array}{c}\text { Number of images retrieved in } \\
\text { existing method }\end{array}$ \\
\hline 1 & 8 & 1 \\
\hline 2 & 22 & 19 \\
\hline 3 & 22 & 20 \\
\hline
\end{tabular}

The above table 1 shows the comparison of number of images retrieved in our proposed and existing method and therefore we need to compute the precision and recall for these retrieved images are as follows

\subsection{Performance evaluation}

The performance of this proposed system is evaluated on different type of query images using Precision, and Recall subsequently these values are compared with the Precision, Recall values of the conventional CBIR system. We have used the Precision and Recall technique, which are described in for evaluating the performance of the proposed content-based image retrieval system.

$$
\begin{aligned}
& : \text { precision }=\frac{\text { Number of retrieved images relevant to the query image }}{\text { Total number of images retrieved }} \\
& : \quad \text { recall }=\frac{\text { Number of retrieved images relevant to the query image }}{\text { Total number of relevant images in the database }}
\end{aligned}
$$

\subsection{Performance Analysis}

The performance of the proposed CBIR system is analyzed with the CBIR system without RRI Library and with the classic CBIR system, which retrieves the features of the whole image.

Table 2: Precision Recall values (proposed work)

\begin{tabular}{|l|c|c|}
\hline Number of retrieval & Precision & Recall \\
\hline First & 0.3333 & 0.32 \\
\hline Second & 0.5945 & 0.88 \\
\hline Third & 0.6667 & 0.88 \\
\hline
\end{tabular}

Table 3: Precision Recall values in (existing method)

\begin{tabular}{|l|c|c|}
\hline Number of retrieval & Precision & Recall \\
\hline First & 0.0416 & 0.04 \\
\hline Second & 0.5135 & 0.76 \\
\hline Third & 0.6061 & 0.8 \\
\hline
\end{tabular}

First retrieval

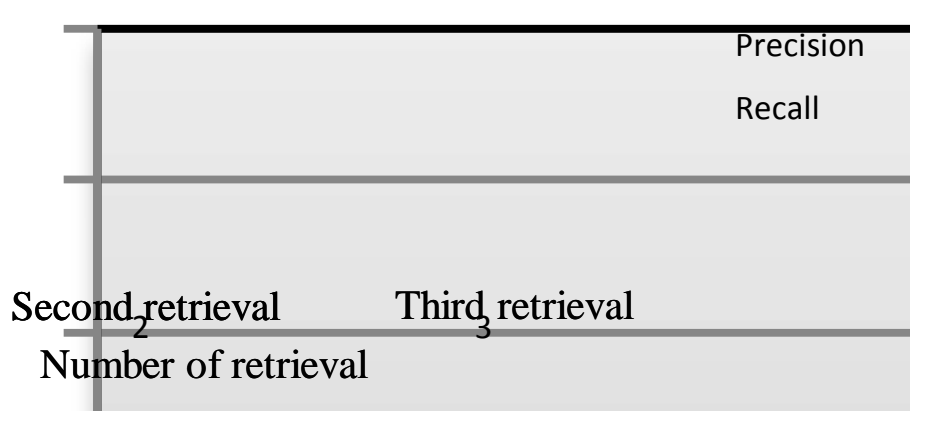




\section{Figure 6: Precision Recall graph (existing method)}

The precision and recall values are calculated using equation (4) and (5). The precision-recall graph of obtained values show that the proposed CBIR system with RRI library claims effectiveness in retrieving images that are most similar to the given query image and its effectiveness increases in each iteration. The fig. 6 and fig. 7 shows the comparison of precision and recall values for the retrieved images in our proposed method and existing method. From the fig. 8 we came to know that the precision and recall of the proposed system increases with each iteration because of the usage of the RRI library. Though the precision and recall value of the proposed system are low in first retrieval its retrieval effectiveness will effectively increase in each of the iteration, which shows the higher performance than the comparing system.

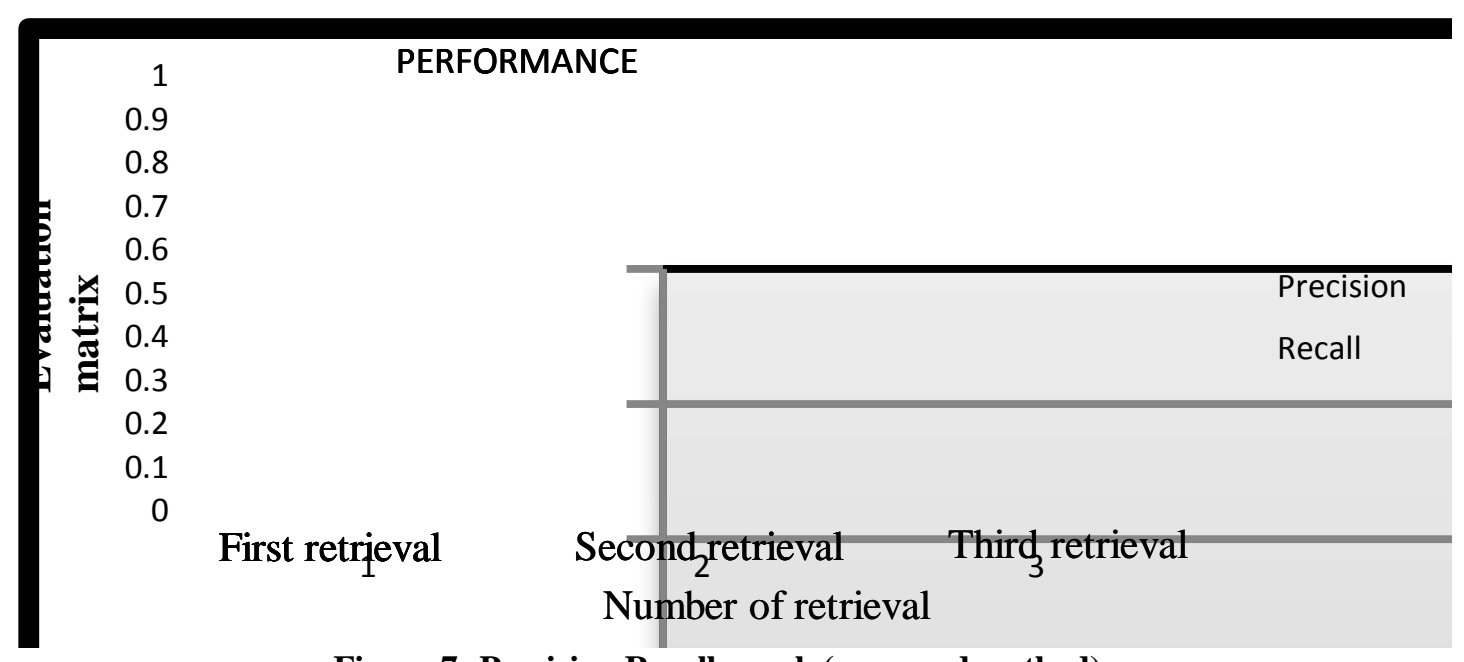

Figure 7: Precision Recall graph (proposed method)

\section{Conclusion}

In this paper, an effective approach to retrieve the images by using the low level and high-level features is proposed. Initially, the low-level features such as color, texture, shape, and homogeneity are extracted from the database images and from the query images. After that, the images which are relevant to the given query image are retrieved from the database based on these low-level features and high level features. By means of the query, keyword, which is a high-level feature, is generated and then by using this query keyword the images, which are relevant to the query keyword, are extracted. It has proved that the performance of the proposed system with homogeneity feature extraction from the RRI library is effective and shows good performance than other conventional CBIR systems. Finally, the proposed system was proved to be effective by querying it with different types of images.

\section{References}

[1] Wang Xiaoling and Xie Kanglin, "Content based Image retrieval incorporating the AHP method", International Journal of Information Technology, Vol.11, No.1, pp.25-37,

[2] Irfan and Sumari, "Automated content based image retrieval using wavelets", International Journal of Computational Intelligence, Vol.1, No.1, pp.1-12, 2004

[3] Chi-Man Pun and Chan-Fong Wong, "Content-Based Image Retrieval Using Rectangular Segmentation", International Journal of Computers, Vol.2, No.1, pp.72-79, 2008

[4] Hiremath and Jagadeesh Pujari, "Content based image Retrieval based on color, texture and shape features using image and its complement", International Journal of Computer science and security, Vol.1, No.4, pp.25-35

[5] Arun and Hema P Menon, "Content Based Medical Image Retrieval by Combining Rotation Invariant Contourlet Features and Fourier Descriptors", International Journal of Recent Trends in Engineering, Vol.2, No.2, pp., November 2009

[6] Srinivasa Rao, Srinivas Kumar and Chandra Mohan, "Content Based Image Retrieval using Exact Legendre moments and support vector machine", The International Journal of Multimedia \& its Applications (IJMA), Vol.2, No.2, pp. 69-79,May 2010

[7] Jalil Abbas, Salman Qadri, Muhammad Idrees, Sarfraz Awan and Naeem Akhtar Khan, "Frame Work for Content Based Image Retrieval(Textual Based) System", Journal of American Science, Vol.6, No.9,pp.704-707, 2010

[8] Christopher C.Yang, "Content based image retrieval: a comparison between query by example and image browsing map approaches", Journal of Information Sciences, Vol.30, No.3, pp.254-267, 2004

[9] Rajshree S.Dubey, Rajnish Choubey and Joy Bhattacharjee, "Multi Feature Content Based Image Retrieval", International Journal on Computer Science and Engineering, Vol.2, No.6, pp.2145-2149, 2010

[10] Nandagopalan, Adiga and Deepak, "A Universal Model for Content-Based Image Retrieval", WASET, Vol.46, No.112, pp.644-647, 2008 
[11] Thomas M. Lehmann,Mark O.Guld, Thomas Deselaers, Daniel Keysers,Henning Schubert, Klaus Spitzer, Hermann Ney and Berthold B. Wein, "Automatic categorization of medical images for content-based retrieval and data mining", Computerized Medical Imaging and Graphics, Vol.29, No.2, pp.143-155, 2005

[12] Serge Belongie, Chad Carson, Hayit Greenspan and Jitendra Malik, "Color and Texture-Based Segmentation using EM and its Application to Content-Based Image Retrieval," In Proceedings of the Sixth International Conference on Computer Vision, Vol. 10, pp. 675-682, Jan 1998.

[13] Jorma Laaksonen, Erkki Oja and Sami Brandt, "Statistical Shape Features in Content-Based Image Retrieval," In Proceedings of the 15th International Conference on Pattern Recognition, Vol.2, pp. 1062 - 1065, Sep 2000

[14] Dengsheng Zhang and Guojun Lu, "A Comparative Study of Curvature Scale Space and Fourier Descriptors for Shape-based Image Retrieval," Journal of Visual Communication and Image Representation, Vol. 14, No. 1, pp. 39-57, Mar 2003

[15] Chia-Hung Wei, Yue Li, Wing Yin Chau and Chang-Tsun Li, "Trademark Image Retrieval Using Synthetic Features for Describing Global Shape and Interior Structure," Journal of Pattern Recognition, Vol. 42, No.3, pp. 386-394, Mar 2009.

[16] Sastry, Saurabah Jain and Ashish Mishra, "Application of 11 norm minimization technique to image retrieval", WASET, Vol.56, No.145, pp.801-804, 2009

[17] Naresh Babu, Sake.Pothalaiah and Ashok Babu, "Image retrieval color, shape and texture features using content based", International Journal of Engineering Science and Technology, Vol.2, No.9, pp.4278-4287,2010

[18] Henning Muller, Nicolas Michoux, David Bandon and Antoine Geissbuhler, "A Review of Content-Based Image Retrieval Systems in Medical Applications-Clinical Benefits and Future Directions", International Journal of Medical Informatics, Vol.73, No.1, pp.1 30,2004

[19] Rong Zhao and William I. Grosky, "Narrowing the Semantic Gap-Improved Text-Based Web Document Retrieval Using Visual Features", IEEE Transactions On Multimedia, Vol.4, No.2, pp.189-200, June 2002

[20] Arnold W.M. Smeulders, Marcel Worring, Simone Santini, Amarnath Gupta and Ramesh Jain, "Content-Based Image Retrieval at the End of the Early Years", IEEE Transactions On Pattern Analysis And Machine Intelligence, Vol.22, No.12, pp.1349-1380, December 2000

[21] Ganapathi Reddy, Babu and Somasekhar, "Image Retrieval by Semantic Indexing", Journal of Theoretical and Applied Information Technology, Vol.5, No.6, pp.745-750, 2005.

[22] Arun Kulkarni, Harikrisha Gunturu and Srikanth Datla, "Association-Based Image Retrieval", WSEAS Transactions on Signal Processing, Vol. 4, No.4, pp.183-189, 2008.

[23] Preeti Aggarwal, Sardana and Gagandeep Jindal, "Content Based Medical Image Retrieval: Theory, Gaps and Future Directions", ICGST-GVIP Journal, Vol.9, No.2, pp. 27-37, 2009.

[24] Wichian Premchaiswadi and Anucha Tungkatsathan, "On-line Content-Based Image Retrieval System using Joint Querying and Relevance Feedback Scheme", WSEAS Transactions on Computers, Vol.9, No.5, pp. 465-474, 2010.

[25] Hui Hui Wang, Dzulkifli Mohamad and Ismail, "Semantic Gap in CBIR: Automatic Objects Spatial Relationships Semantic Extraction and Representation", International Journal of Image Processing (IJIP), Vol.4, No.3, 192-204, 2010.

[26] Jianhua Wu, Zhaorong Wei and Youli Chang, "Color and Texture Feature for Content Based Image Retrieval", International Journal of Digital Content Technology and its Applications, Vol.4, No.3, pp. 43-49, 2010

[27] Sankara Rao, Vamsidhar, Samuel Vara Prasad Raju, Ravikanth SAtapati and Varma,"An Approach For Cbir System Through Multi Layer Neural Network", International Journal of Engineering Science and Technology, Vol.2, No.4, pp.559-563, 2010 